\title{
New Phase Shifting Algorithms Insensitive to Linear Phase Shift Errors
}

J. Novák

This article describes and analyses multistep algorithms for evaluating of the wave field phase in interferometric measurements using the phase shifting technique. New phase shifting algorithms are proposed, with a constant but arbitrary phase shift between captured frames of the intensity of the interference field. The phase evaluation process then does not depend on linear phase shift errors. A big advantage of the described algorithms is their ability to determine the phase shift value at every point of the detector plane. A detailed analysis of these algorithms with respect to main factors that affect interferometric measurements is then carried out. The dependency of these algorithms on phase shift values is also studied several phase calculation algorithms are proposed. These are compared with respect to the resulting phase errors.

Keywords: noncontact deformation measurement, phase calculation algorithms, error analysis.

\section{Introduction}

The problems of techniques for automatic evaluation of interferometric measurements are much studied nowadays, because of the rapid development of digital interferometric techniques and their applications in industrial practice [1-10]. Depending on the specific character of the measurement problem to be solved, various phase evaluation methods can be used [11-19]. Some of these methods use only one intensity frame, i.e. an interferogram, to determine the phase values, while other methods need to record several interferograms to calculate the phase unambiguously. One of the most popular and most accurate methods for phase evaluation is the phase shifting technique [2,4,16-19]. The phase shifting technique was first applied for analysis of interferometric measurements in the field of classical two beam interferometry, and nowadays it is the most widely used technique for evaluating of interference fields in many areas of science and engineering. The method is based on evaluating phase values from several phase modulated measurements of the intensity of the interference field. It is necessary to carry out at least three phase shifted intensity measurements in order to make an unambiguous and very accurate determination of the phase at every point of the detector plane. The phase shifting technique offers fully automatic calculation of the phase difference between two coherent wave fields that interfere. There exist many phase shifting algorithms for phase calculation that differ in the number of phase steps, in phase shift values between captured intensity frames, and in their sensitivity to factors that affect interferometric measurements [2, 16, 18, 20, 21]. The following text describes new proposed algorithms that are insensitive to phase shift miscalibration, and a complex error analysis of these phase calculation algorithms is performed.

\section{Phase shifting technique for analysis of interferometric measurements}

The general principle of most interferometric measurements as follows. Two light beams (reference and object) interfere after an interaction of the object beam with the measured object, i.e. the beam is transmitted or reflected by the object. The distribution of the intensity of the interference field is then detected, e.g. using a photographic plate, CCD camera, etc. The phase difference between the reference and the object beam, which is related to the measured quantity, e.g. displacement, can be determined using various phase calculation techniques.

The phase shifting technique is based on an evaluation of the phase of the interference signal using phase modulation of this interference signal. The intensity distribution $I$ of the detected phase modulated interference signal at some point $(x, y)$ of the detector plane is given by $[2,4,22]$

$$
\begin{aligned}
I_{i}(x, y) & =A(x, y)+B(x, y) \cos \left[\Delta \varphi(x, y)+\psi_{i}\right] \\
i & =1, \ldots, N \quad N \geq 3,
\end{aligned}
$$

where $A$ is the function of the background intensity, $B$ is the function of the amplitude modulation, $\Delta \varphi$ is the phase difference of the wave fields that interfere, and $\psi_{i}$ is the phase shift of the $i$-th intensity measurement. To determine phase values $\Delta \varphi$ it is necessary to solve nonlinear equations (1) for every point $(x, y)$ of the detected interference pattern. If the phase shift values $\psi_{\mathrm{i}}$ are properly chosen in advance, there remain only three unknowns $A, B$ and $\Delta \varphi$ that must be determined from the recorded intensity values [2, 4, 11]. We must carry out at least $N=3$ intensity measurements with known phase shift values $\psi_{\mathrm{i}}$ to calculate phase $\Delta \varphi$.

\section{New phase shifting algorithms}

We will now show several new multistep phase shifting algorithms that are insensitive to linear phase shift errors. We will consider constant but unknown phase shift values $\psi$ between recorded images of the intensity of the observed interference field. Then it is necessary to capture at least four interferograms, because the interference equation (1) consists of four unknowns A, B, $\psi$ and $\Delta \varphi$. From the above-mentioned assumption we can derive phase calculation algorithms that are insensitive to linear phase shift errors. The very well known Carré algorithm is a phase shifting algorithm of this type $[8,11,23]$. For the intensity at every point of the recorded interferogram we can write

$$
I_{k}=A+B \cos \left[\Delta \varphi+\frac{(2 k-N-1)}{2} \psi\right], \quad k=1, \ldots, N
$$


where $\psi$ is the constant phase shift between captured intensity frames, $A$ is the mean intensity, and $B$ is the modulation of the interference signal. Using very well known trigonometric relations we obtain the following equations for the phase values $\Delta \varphi$ (Table 1).

From these phase calculation algorithms it is evident that firstly the phase values $\Delta \varphi_{W} \in[-\pi, \pi]$ must be determined and then the discontinuities in the phase data can be properly unwrapped using suitable phase unwrapping procedures [24-26]. For unambiguous determination of the wrapped phase values $\Delta \varphi_{\mathrm{W}}$ it is necessary to find for the described algorithms appropriate expressions proportional to functions $\sin \Delta \varphi$ and $\cos \Delta \varphi$. Such expressions are shown in Table 2. Then according to the sign of the expressions we can determine the wrapped phase values $\Delta \varphi_{W} \in[-\pi, \pi]$.

A big advantage of this type of phase calculation algorithm with unknown value of the phase shift is that we can calculate the phase shift at all points $(x, y)$ of the detector in order to control the distribution of the phase shift over the interferogram. These phase evaluation algorithms are not

Table 1

\section{Agorithm}

A1

A2

A3

$$
\tan \Delta \varphi=\frac{\left(I_{1}-I_{5}-2 I_{2}+2 I_{4}\right)}{\sqrt{4\left(I_{2}+I_{4}-2 I_{3}\right)^{2}-\left(I_{1}+I_{5}-2 I_{2}-2 I_{4}+2 I_{3}\right)^{2}}}
$$

A4

$$
\tan \Delta \varphi=\frac{\sqrt{4\left(I_{2}-I_{5}\right)^{2}-\left[\left(I_{3}-I_{4}\right)+\left(I_{1}-I_{6}\right)\right]^{2}}}{\left(I_{3}+I_{4}\right)-\left(I_{1}+I_{6}\right)}
$$

A5

$$
\tan \Delta \varphi=\sqrt{\frac{2\left(I_{2}-I_{5}\right)-\left(I_{3}-I_{4}\right)-\left(I_{1}-I_{6}\right)}{2\left(I_{2}-I_{5}\right)+\left(I_{3}-I_{4}\right)+\left(I_{1}-I_{6}\right)}} \frac{I_{3}-I_{4}-I_{1}+I_{6}}{I_{3}+I_{4}+I_{1}+I_{6}-2\left(I_{2}+I_{5}\right)}
$$

$$
\tan \Delta \varphi=\frac{\left(I_{3}-I_{5}\right)}{\left(2 I_{4}-I_{2}-I_{6}\right)} \frac{\sqrt{4\left(I_{2}-I_{6}\right)^{2}-\left[\left(I_{3}-I_{5}\right)+\left(I_{1}-I_{7}\right)\right]^{2}}}{\left(I_{2}-I_{6}\right)}
$$

$$
\tan \Delta \varphi=\frac{\sqrt{\left(I_{3}-I_{5}\right)\left[3\left(I_{3}-I_{5}\right)-\left(I_{1}-I_{7}\right)\right]}}{\left(2 I_{4}-I_{2}-I_{6}\right)}
$$

\begin{tabular}{ccc}
\hline Algorithm & Expressions $\sim \sin \Delta \varphi$ & Expressions $\sim \cos \Delta \varphi$ \\
\hline A1 & {$\left[I_{2}-I_{3}\right]$} & {$\left[\left(I_{2}+I_{3}\right)-\left(I_{1}+I_{4}\right)\right]$} \\
\hline A2 & {$\left[I_{2}-I_{4}\right]$} & {$\left[2 I_{3}-I_{1}-I_{5}\right]$} \\
\hline A3 & {$\left[I_{1}-I_{5}-2 I_{2}+2 I_{4}\right]$} & {$\left[I_{2}+I_{4}-2 I_{3}\right]$} \\
\hline A4 & {$\left[\left(I_{3}-I_{4}\right)+\left(I_{1}-I_{6}\right)\right]$} & {$\left[\left(I_{3}+I_{4}\right)-\left(I_{1}+I_{6}\right)\right]$} \\
\hline A5 & {$\left[\left(I_{3}-I_{4}\right)-\left(I_{1}-I_{6}\right)\right]$} & {$\left[I_{3}+I_{4}+I_{1}+I_{6}-2\left(I_{2}+I_{5}\right)\right]$} \\
\hline A6 & {$\left[\left(I_{3}-I_{5}\right)+\left(I_{1}-I_{7}\right)\right]$} & {$\left[\left(I_{2}+I_{6}\right)-2 I_{4}\right]$} \\
\hline A7 & {$\left[3\left(I_{3}-I_{5}\right)-\left(I_{1}-I_{7}\right)\right]$} & {$\left[2 I_{4}-\left(I_{2}+I_{6}\right)\right]$} \\
\hline
\end{tabular}


affected by miscalibration of the phase shift device and they can be used if the phase shift is nonuniformly distributed over the area of the detector.

\section{Analysis of phase shifting algorithms}

The overall accuracy of interferometric measuring techniques is determined by systematic and random errors during the measurement process. There are many potential factors that may influence the measurement accuracy. For analysis of the influence of the most important factors that have a negative effect on the accuracy of interferometric measurement techniques, a mathematical model has been proposed that enables the accuracy and stability of phase shifting algorithms to be analysed with respect to chosen parameters of the influencing factors [18]. This relationship has been studied because the stability and accuracy of the described phase evaluation algorithms with unknown phase shifts also depends on the phase shift value itself. Performing the computer analysis, the optimal phase shift values were obtained for all algorithms. The given phase shifting algorithms have the lowest phase errors for these values. The accuracy and sensitivity of the phase shifting algorithms was evaluated on the basis of the mean phase error $\overline{\delta(\Delta \varphi)}$. The mean phase error is given by

$$
\overline{\delta(\Delta \varphi)}=\frac{\sum_{k=1}^{M} \delta(\Delta \varphi)_{k}}{M},
$$

where $\delta(\Delta \varphi)_{k}$ is $k$-th simulation of the phase error $\delta(\Delta \varphi)$ for given measurement conditions, and $M$ is the number of simulation cycles. The modelled function $\overline{\delta(\Delta \varphi)}$ that is shown in Fig. 1 expresses the obtained accuracy for arbitrary phase values $\Delta \varphi$ and the chosen phase shift value $\psi$.
The optimal phase shift value $\psi_{\text {opt }}$ is found, where the phase error function $\overline{\delta(\Delta \varphi)}$ reaches its minimum. These values were obtained from the simulated relationship using a polynomial approximation near the minima of these functions. The resulting phase shift values $\psi_{\text {opt }}$ are shown in Table 3.

Table 3

\begin{tabular}{|c|c|c|c|}
\hline Algorithm & $\begin{array}{c}\text { Number of } \\
\text { steps } N\end{array}$ & $\begin{array}{c}\text { Phase shift } \\
\psi_{\text {opt }}[\mathrm{rad}]\end{array}$ & $\begin{array}{c}\text { Phase shift } \\
\psi_{\text {opt }}\left[{ }^{\circ}\right]\end{array}$ \\
\hline A1 & 4 & $0.61 \pi$ & 110 \\
\hline A2 & 5 & $0.50 \pi$ & 90 \\
\hline A3 & 5 & $0.67 \pi$ & 121 \\
\hline A4 & 6 & $0.38 \pi$ & 69 \\
\hline A5 & 6 & $0.54 \pi$ & 97 \\
\hline A6 & 7 & $0.34 \pi$ & 61 \\
\hline A7 & 7 & $0.50 \pi$ & 90 \\
\hline
\end{tabular}

Fig. 1 shows clearly that algorithms A1, A2, A3 and A7 have a relatively wide range of phase shift values, where the phase error is approximately the same as the optimal value. Therefore these phase-shifting algorithms are not too sensitive to a change in the optimal phase shift value.

With calculated optimal phase shift values for every algorithm a complex error analysis was carried out. The influence of errors of the phase shifting device and the detector were simulated using an appropriate model of these errors [18]. Figure 2 shows the relationship between the calculated phase error $\delta(\Delta \varphi)$ and the phase values $\Delta \varphi$ from the range $(-\pi, \pi)$, which enables a comparison of the accuracy and stability of phase calculation using a particular phase-shifting algorithm.

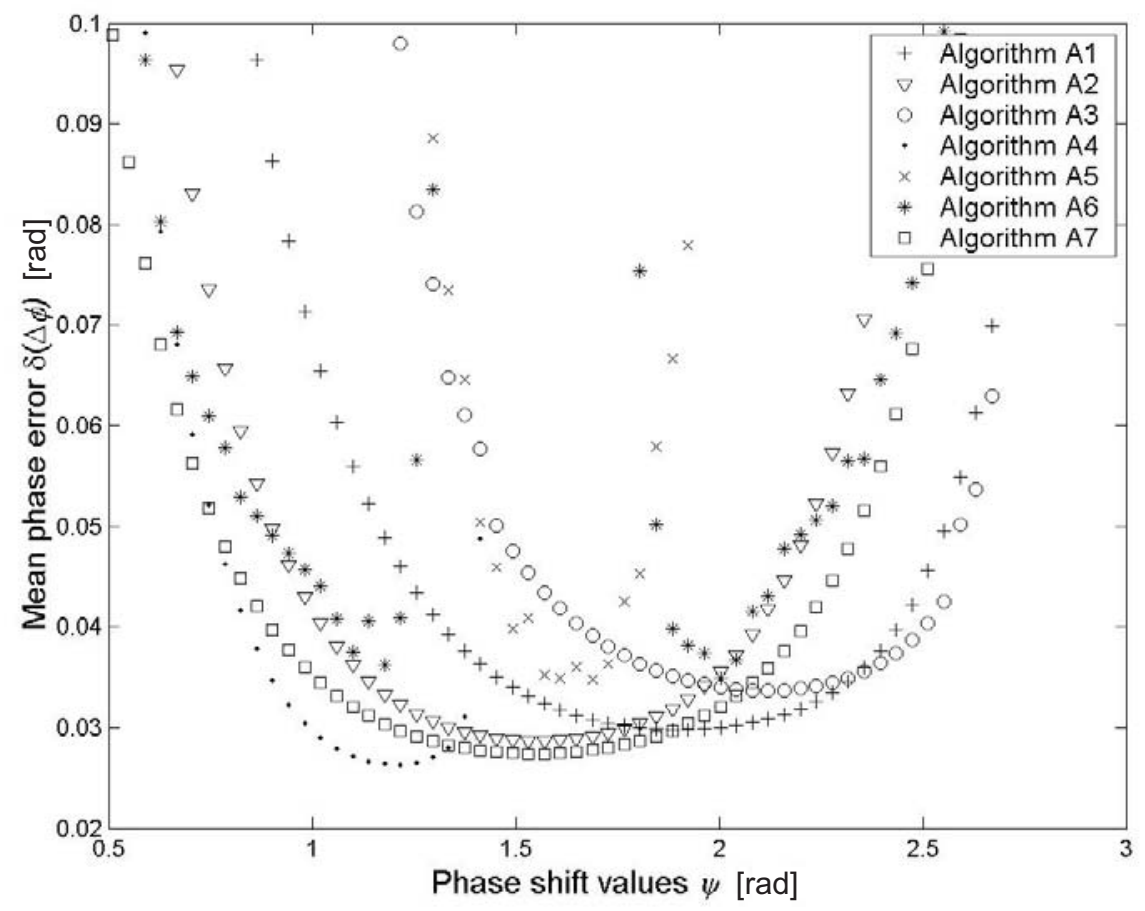

Fig. 1: Optimal phase shift values 




Fig. 2: Relationship between phase error $\delta(\Delta \varphi)$ and phase values $\Delta \varphi$

Further, a study was made of the properties of the phase shifting algorithms with respect to the change of parameters simulating the real nonlinearities of the phase shifting device and detector. With respect to the insensitivity of the phase measuring algorithms to linear phase shift errors, we studied only the dependency of the phase errors on the second order errors of the devices. The dependency of the phase error $\delta(\Delta \varphi)$ of the phase evaluation on the nonlinear behaviour of the phase shifting device is shown in figure 3 for all compared algorithms. Figure 4 demonstrates the dependency of the phase error on the nonlinear response of the detector of the intensity.

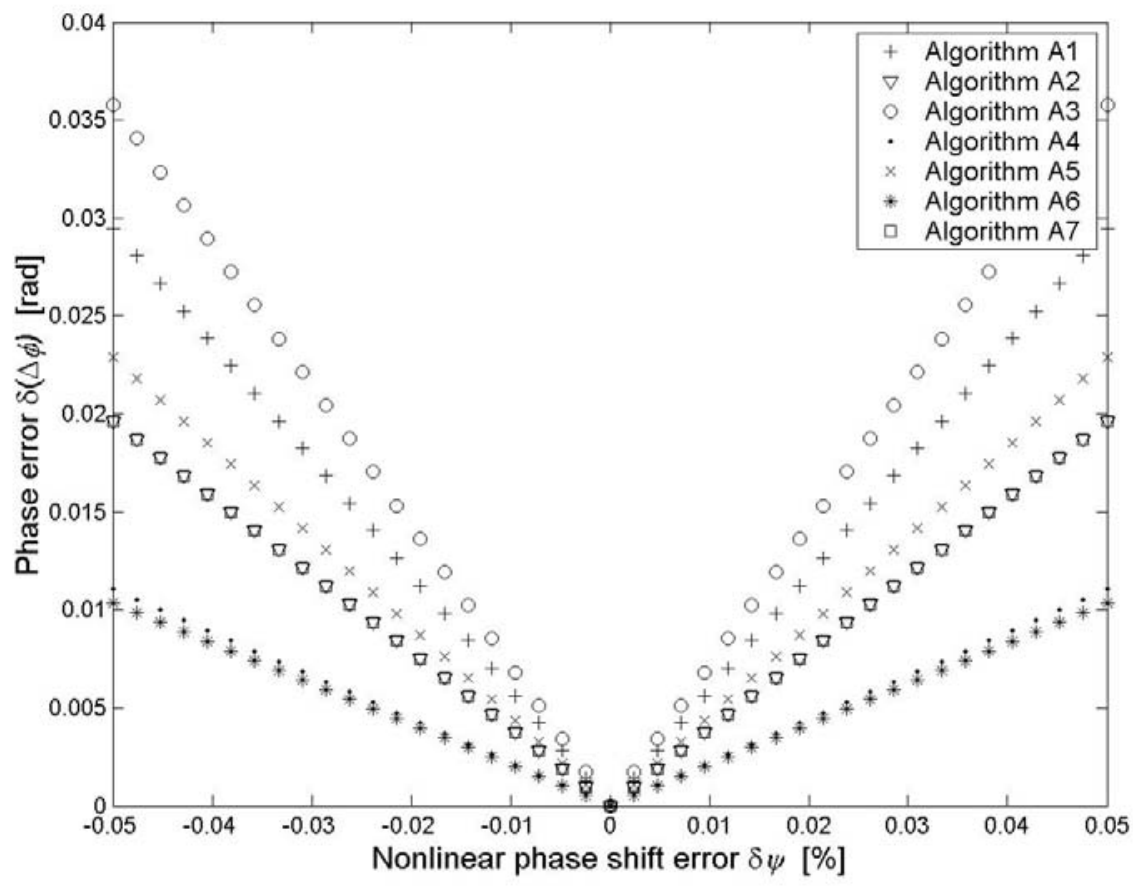

Fig. 3: Effect of nonlinear phase shift errors 


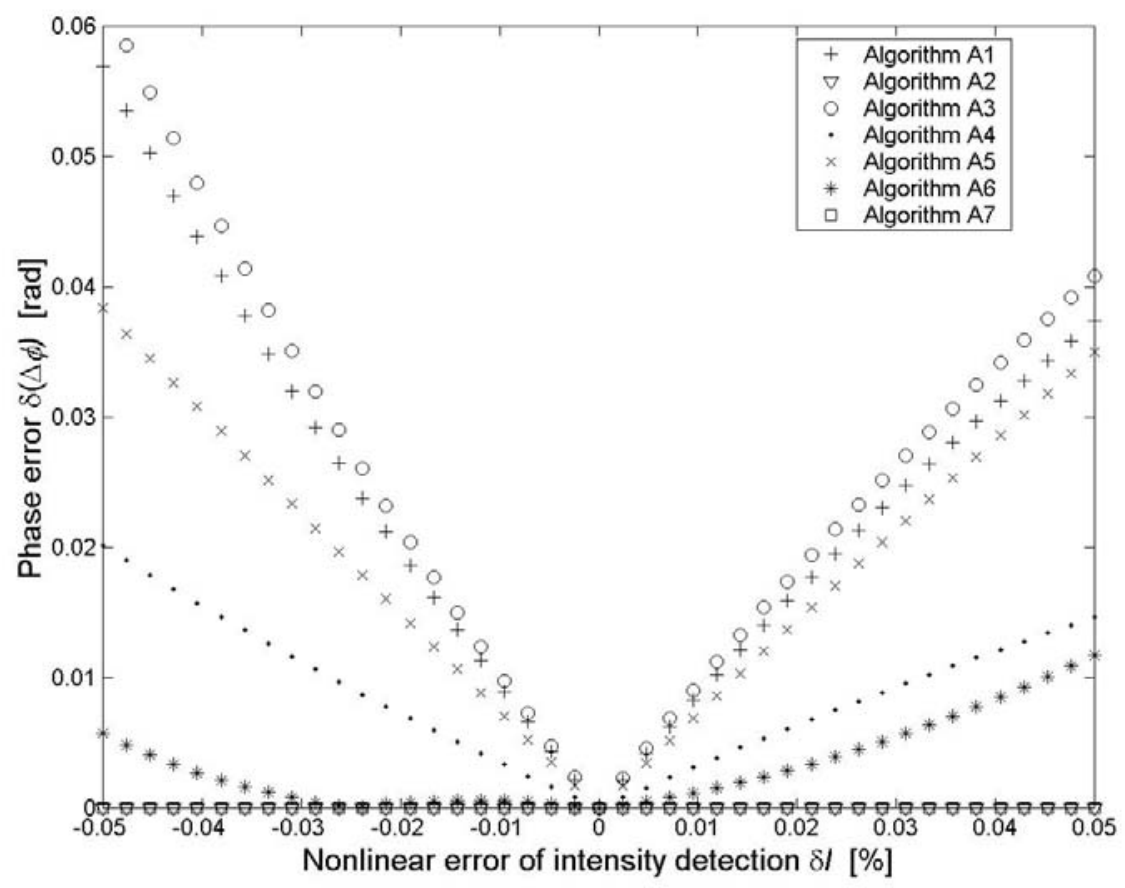

Fig. 4: Effect of nonlinear response of detector

Finally, the computing time for phase shifting algorithms A1-A7 was determined. The relative computing time was calculated as the ratio between the computing time for a given algorithm and the minimum computing time of all the compared algorithms (see table 4).

Table 4

\begin{tabular}{|c|c|}
\hline Algorithm & $\begin{array}{r}\text { Relative computing } \\
\text { time }[\%]\end{array}$ \\
\hline A1 & 108 \\
\hline A2 & 100 \\
\hline A3 & 140 \\
\hline A4 & 101 \\
\hline A5 & 146 \\
\hline A6 & 130 \\
\hline A7 & 102 \\
\hline
\end{tabular}

On the basis of the error analysis we can conclude that the most accurate algorithms are the five-step algorithm A2, the six-step algorithm A4, and the seven-step algorithm A7. However, the six-step algorithm A5 is the least accurate and its computing time is the longest. The computing time depends on the number of mathematical operations. It can be seen that the computing time does not depend directly on the increasing number of steps $N$. For example, the computing time of algorithms A2, A4 and A7 is practically the same. Algorithms A2 and A7 seem to be particularly accurate, having stable properties for a wide range of phase step values.

\section{Conclusion}

This paper deals with algorithms for phase calculation in interferometric measurement methods using the phase shifting technique. It describes new multistep phase shifting algorithms with constant but unknown phase steps between the intensity frames. These phase evaluation algorithms are not sensitive to phase shift miscalibration and enable calculation of the actual phase shift value at any point of the detector plane during the measurement process. With these algorithms, the phase shifting device can be calibrated and measurement can be carried out if the phase shift is not uniformly distributed over the detector area. Phase calculation algorithms with unknown phase shifts can be applied for any measurement that uses the phase shifting technique for measurement evaluation. The accuracy and stability with respect to the initial phase shift values of all described algorithms were studied, and optimal phase shift values for particular algorithms were determined in order to ensure the lowest possible phase errors of the phase calculation algorithms. On the basis of the proposed model, an analysis was performed of the influence of the most important parameters that have a negative effect on the overall accuracy and stability of phase calculation algorithms, and these algorithms were compared.

\section{Acknowledgement}

This work was supported by grant No. 103/02/0357 of the Grant Agency of the Czech Republic.

\section{References}

[1] Cloud, G.: Optical methods of engineering analysis. Cambridge: Cambridge Univ. Press, 1998. 
[2] Malacara, D.: Optical shop testing. New York: John Wiley \& Sons, 1992.

[3] Rastogi, P. K.: Digital speckle pattern interferometry and related techniques. New York: John Wiley \& Sons, 2001.

[4] Kreis, T.: Holographic interferometry: Principles and methods. Berlin: Akademie Verlag, 1996.

[5] Rastogi, P. K.: Handbook of optical metrology. Boston: Artech House Publishing, 1997.

[6] Jacquot, P., Fournier, J. M.,(ed.): Interferometry in speckle light: Theory and applications. Berlin: Springer Verlag, 2000 .

[7] Osten, W., Jüptner, W., (ed.): Fringe 2001: The $4^{\text {th }}$ international workshop on automatic processing of fringe patterns. Paris: Elsevier, 2001.

[8] Osten, W., Jüptner, W., Kujawinska, M. (ed.): Optical measurement systems for industrial Inspection II. SPIE Proceedings, Vol. 4398, Washington: SPIE, 2001.

[9] Kobayashi, A. S. (ed.): Handbook of experimental mechanics. New Jersey: Prentice Hall, 1987.

[10] Rastogi, P. K., Inaudi, D.: Trends in optical non-destructive testing and inspection. Amsterdam: Elsevier, 2000.

[11] Malacara, D., Servin, M., Malacara, Z.: Interferogram analysis for optical testing. New York: Marcel Dekker, 1998.

[12] Yatagai, T.: Automatic fringe analysis using digital image processing techniques. Optical Engineering, Vol. 21, 1982, p. 432.

[13] Novak, J.: Fringe tracing technique in the process of optical testing. Physical and material engineering 2002, Prague, CTU 2002.

[14] Takeda, M., Ina, H., Kobayashi, S.: Fourier-transform method of fringe-pattern analysis for computer-based topography and interferometry. J. Opt. Soc. Am, Vol. 72, No. 1, 1982, p.156.

[15] Kujawinska, M., Wójciak, M.: High accuracy Fourier transform fringe pattern analysis. Optics and Lasers in Engineering, Vol. 14, 1991, p. 325-339.

[16] Creath, K.: Phase-measurement interferometry techniques. Progress in optics, Vol. XXVI, Amsterdam: Elsevier Science, 1988.
[17] Robinson, D. W., Reid, G. T.: Interferogram analysis: Digital fringe pattern measurement techniques. Bristol: Institute of Physics Publishing, 1993.

[18] Novak, J.: Computer simulation of phase evaluation process with phase shifting technique. Physical and material engineering, Prague: CTU 2002, p. 87-88.

[19] Miks, A., Novak, J.: Application of multi-step algorithms for deformation measurement. SPIE Proceedings, Vol. 4398, Washington: SPIE, 2001, p. 280-288.

[20] Novak, J.: Error analysis of three-frame algorithms for evaluation of deformations. Interferometry of speckle light: Theory and applications, Berlin: Springer Verlag, 2000, p. 439-444.

[21] Novak, J.: Error analysis for deformation measurement with electro-optic holography. Fine mechanics and optics, Vol. 6, 2000, p. 166.

[22] Miks, A.: Applied optics 10. (in Czech), Prague: CTU Publishing House, 2000.

[23] Carré, P.: Installation et Utilisation du Comparateur Photoelectrique et Interferential du Bureau International des Poids de Mesures. Metrologia Vol. 2, 1966, p. 13-23

[24] Ghiglia, D. C., Pritt, M. D.: Two-dimensional phase unwrapping: Theory, algorithms and software. New York: John Wiley \& Sons, 1998.

[25] Novak, J.: Methods for 2-D phase unwrapping. In MATLAB 2001 Proceedings, Prague: VŠCHT Publishing House, 2001.

[26] Gutmann, B., Weber, H.: Phase unwrapping with the branch-cut method: clustering of discontinuity sources and reverse simulated annealing. Applied Optics, Vol. 38, No. 26, 1999, p. 5577.

Ing. Jiří Novák, Ph.D.

phone: +420224354435

fax: +420233333226

e-mail:novakji@fsv.cvut.cz

Department of Physics

Czech Technical University in Prague

Faculty of Civil Engineering

Thákurova 7

16629 Prague 6, Czech Republic 